\title{
EDitorial
}

\section{Is there any relationship between Neuroscience and Psychiatry}

\author{
Luis C. Farhat, Marcelo Q. Hoexter, Euripedes C. Miguel
}

Psychiatry has always been considered a separate field of medicine among other medical specialties. Unlike Cardiology or Oncology, diagnoses of psychiatric disorders are made entirely based on patients' presenting signs and symptoms. There are no sources of external validity (e.g. laboratory exam, imaging findings) that can be employed reliably to identify individuals with psychiatric disorders. Additionally, our understanding of the pathophysiologic processes underlying psychiatric phenomena currently lags behind that of other pathological processes concerning to other medical specialties such as Cardiology and Oncology. Our poor understanding of psychiatric disease is probably one of the main reasons why treatments of psychiatric disorders are non-specific and, usually, non-efficient.

Yet, recent advancements in neuroscience have contributed to clarify the neurobiological underpinnings of psychiatric phenomena. Psychiatric disorders are increasingly recognized as disorders of the brain, and increasing research is carried out to identify potential genetic, neuroanatomical and neurofunctional correlates that reliably predict psychiatric diagnoses, prognosis and patterns of response to different treatments. In that way, there is increasing optimism among clinicians and researchers of the field that mental health problems will soon be managed better than nowadays.

In this issue of Revista de Medicina, we present 7 review articles which highlight the advancements in neuroscience in Psychiatry. Novic and Ross initially describes how integrating a modern neuroscience perspective into medical education allows students to conceptualize psychiatric patients in a way that promotes empathy and enhances patient care. Leckman and colleagues discuss the role of the 'immune system' in normal brain development, as well as in the pathobiology of neurodevelopmental and neuropsychiatric disorders. Murakami and colleagues discuss how neuroimaging and electroencephalographic techniques can be employed to investigate neural circuits in psychiatric disorders. Carui and colleagues and Farhat and colleagues discuss current-models of our understanding of addictive behaviors and obsessive-compulsive disorder, respectively. Lastly, Suen and colleagues discuss what non-invasive brain stimulation techniques are and how they can be employed in the treatment of psychiatric disorders. Besides the 7 review articles, Belkovsky and colleagues report interesting findings regarding interthalamic adhesions and their relationship with 
Farhat LC, et al. Is there any relationship between neuroscience and psychiatry.

\section{Alzheimer's Diseases.}

Neuroscience can contribute significantly to our understanding of psychiatric disorders, and, hopefully, will lead to improvements in treatments and patient care. Currently, neuroscientific perspectives of psychiatric disorders are yet under considerable research and relatively little benefit has been translated to clinical practice in Psychiatry. Nonetheless, psychiatrists are still optimistic that achievements in neuroscience will eventually lead to improvements in how psychiatric patients are treated. We hope this issue provides an insight to its reader about the potential neuroscience has in improving current practices in Psychiatry. 